\title{
Heavy-hadron interactions from Lattice QCD
}

\author{
Daniel Mohler ${ }^{1,2, *}$ \\ ${ }^{1}$ Helmholtz-Institut Mainz, 55099 Mainz, Germany \\ ${ }^{2}$ Johannes Gutenberg Universität Mainz, 55099 Mainz, Germany
}

\begin{abstract}
I review recent progress in heavy hadron spectroscopy and interactions from ab-initio Lattice QCD calculations. After motivating lattice spectroscopy calculations for heavy-hadrons by contrasting recent $\mathrm{LHCb}$ results for charmed and doubly-charmed baryons with lattice predictions, selected results from scattering calculations for heavy-light mesons and for charmonia are presented. I close with a discussion of recent Lattice QCD predictions of explicitly exotic doubly-heavy states.
\end{abstract}

\section{Introduction}

In Lattice Quantum Chromodynamics, the action of QCD is regularized by a 4-dimensional Euclidean space-time lattice. It is an ab-initio, non-perturbative approach which can be improved systematically and thereby allows for a full control of systematic uncertainties. While calculations on fine lattices with dynamical quarks require large computational capabilities, progress in both hardware and algorithms have allowed for modern calculations to determine quantities of phenomenological relevance from QCD. An example is given by the flavor physics results collected in the FLAG review [1]. Most Lattice QCD calculations work in the isospin limit, and the light up and down as well as the heavier strange (referred to as $2+1$ flavor simulations) or even light, strange and charm quarks (referred to as $2+1+1$ flavor simulations) are included in the creation of gauge configurations (dynamical simulation). While many lattice simulations still employ heavier than physical light-quark masses corresponding to heavier than physical pions, calculations at physical pion masses are now feasible and many results in the FLAG review already result from ensembles with close-to-physical pion masses.

More recently, the isospin splittings from both non-degenerate up- and down-quark masses, as well as from their different charge under QED have been taken into account. An impressive example is provided in Figure 1 from a simulation by the Budapest-MarseilleWuppertal (BMW) collaboration [2], which shows Isospin splittings for various hadrons determined from a dynamical QCD+QED simulation.

In this review we will focus on recent progress in the determination of the heavy-hadron spectrum from Lattice QCD, with particular emphasis on extracting resonances and bound states from heavy-hadron scattering in a finite volume. The latter is based on a finite-volume technique first worked out by Lüscher [3, 4], that relates the energy shifts in a hypercubic finite volume to infinite-volume scattering amplitudes. In its original form, the phase shift for elastic scattering is inferred from rest-frame calculations in multiple spatial volumes $L^{3}$.

*e-mail: damohler@uni-mainz.de 


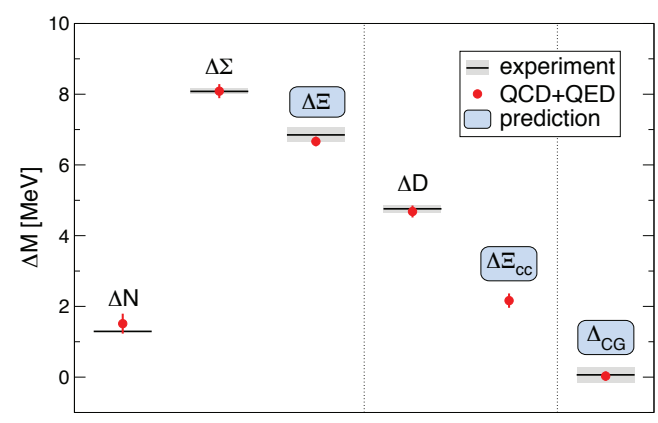

Figure 1. Isospin splitting from $\mathrm{QCD}+\mathrm{QED}$ by the BMW collaboration. The error bars denote the full uncertainty estimate while the black bars and shaded regions show the experiment values. The splitting $\Delta \Xi_{c c}$ is of importance for the discussion in Section 2. Figure taken from [2]

This original method has been generalized in many ways. One kind of improvement focuses on obtaining more information from available lattices, by making use of calculations in both the rest frame and in a number of moving frames (for a first generalization see [5]). More important, the method has also been generalized to allow for coupled-channel scattering with an arbitrary number of two-hadron channels, for the treatment of $2 \leftrightarrow 1$ and $2 \leftrightarrow 2$ transitions (such as $\pi \pi \rightarrow \pi \gamma^{*}$ ), and to allow for 3-particle scattering. For an account of these generalizations please refer to a recent review [6] and to the references therein.

One requirement for successfully applying these finite volume methods to QCD is the availability of an efficient way to calculate all (valence) quark-line diagrams. In particular, the channels with attractive interaction, which are most relevant phenomenologically, typically involve diagrams where quarks propagate from a given timeslice back to the same timeslice. (The simplest of these would be the "disconnected diagrams" arising in the case of an isoscalar meson.) Recent techniques such as the Distillation [7] and Stochastic Distillation (or LapH) [8] methods allow the determination of these diagrams, while at the same time offering a large freedom for the construction of the lattice operators used for the creation and annihilation of the hadrons. For heavy quarks, another important ingredient is the development of improved heavy-quark actions (see for example $[9,10]$ ), which allow for reasonably small discretization effects on currently available lattices with a lattice spacing $a$ of roughly 0.05 to $0.12 \mathrm{fm}$.

In section 2, I will provide a motivation for modern lattice spectroscopy calculations, by taking a look at existing lattice calculations for heavy baryons containing charm quarks, and contrasting these calculations with recent results from the LHCb experiment. In section 3, I will provide an overview of lattice calculations of heavy-light mesons, where a number of results for bound-states and resonances in meson-meson scattering exist [11-16]. In section 4 , exploratory results for charmonium and charmonium-like states are presented. Before concluding, I present an account of Lattice QCD predictions for explicitly exotic doublyheavy hadrons predicted by several groups in Section 5. It should be noted that many of the results covered in this review are exploratory in nature, i.e. not all systematic uncertainties have been investigated. In particular some results discussed are obtained at unphysical lightquark masses, and many of the studies discussed did not perform an extrapolation to zero lattice spacing. As such most results are qualitative in nature. In addition, these results provide an outlook on future Lattice QCD results. 


\section{Charmed heavy baryons}

Recently, the LHCb collaboration observed a doubly-charmed Cascade baryon $\Xi_{c c}^{++}$[17]. They observe a clear signal in data from LHC runs at both $13 \mathrm{TeV}$ and $8 \mathrm{TeV}$, and determine the mass of the hadron to be $3621.40 \pm 0.72 \pm 0.27 \pm 0.14 \mathrm{MeV}$. A number of years ago, an isospin partner to this state has been claimed by the SELEX collaboration $[18,19]^{1}$. The $\Xi_{c c}$ state from SELEX has mass of approximately $3519 \mathrm{MeV}[18,19]$, and was not seen in other subsequent experiments [21-25], casting some doubt on this result.

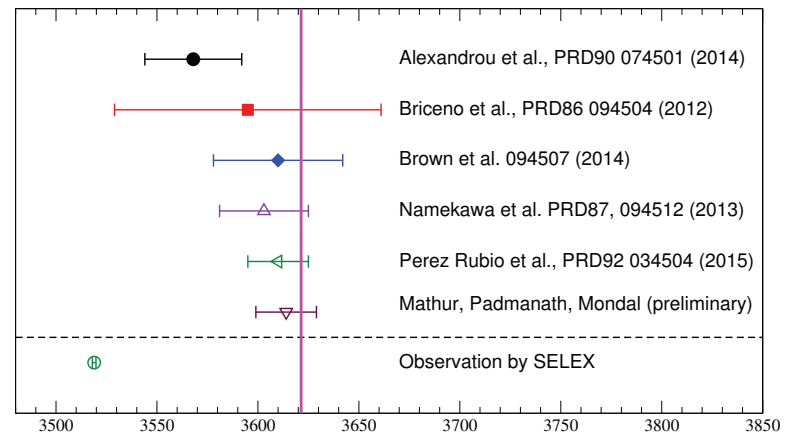

Figure 2. Overview of selected Lattice QCD predictions for the $\Xi_{c c}$ in the isospin limit. Full symbols are chosen for calculations with good control of all relevant systematic uncertainties, while open symbols indicate that the results are results at a single (but reasonably fine) lattice spacing rather than continuum results. The results are compared to the state observed by SELEX $[18,19]$ (green circle) and to the recent observation by LHCb [17] (magenta line).

Before the observation by the LHCb collaboration, a number of lattice calculations yielded predictions for the mass of $\Xi_{c c}$ in the isospin limit. The most advanced of these determinations [26-31] are collected in Figure 2, together with the state claimed by SELEX (green circle) and the observation by LHCb (magenta vertical band). While having a sizeable uncertainty, which largely stems from the limited set of gauge configurations, the various lattice determinations agree within uncertainties and the more precise determinations show considerable tension with the observation by SELEX, but agree quite well with the observed mass from LHCb. Beyond these more comprehensive calculations, a host of older predictions with a somewhat less well determined systematic uncertainty exist (see for example [32] for an impressive early prediction). Note that in addition to this doubly charmed baryon, many other charmed baryons are predicted in the same literature, some of which can likely be observed by $\mathrm{LHCb}$ in the future.

In another recent observation by LHCb, 5 narrow charmed $\Omega_{c}$ baryons have been discovered [33]. Figure 3 shows the discovery plot from LHCb with clearly observed and well separated peaks. While the spin-parity of these states has not yet been determined from experiment, the fact that the observed baryons seem to be quite narrow allows for a qualitative comparison to finite volume energies determined from 3-quark Euclidean space correlation functions calculated on the lattice. ${ }^{2}$

\footnotetext{
${ }^{1}$ Note that this Isospin splitting has been determined from Lattice QCD in [2] and has been found to be quite small, as one would in general expect. For a discussion of doubly-heavy baryon isospin splittings also see [20]. From a theoretical point of view it is therefore essentially impossible that this state could be an isospin partner.

${ }^{2}$ This approach essentially treats these hadrons as stable under the strong interaction, and will therefore only be applicable to very narrow resonances, as the naive uncertainty estimate of such a prediction is of the order of the width of the resonance.
} 


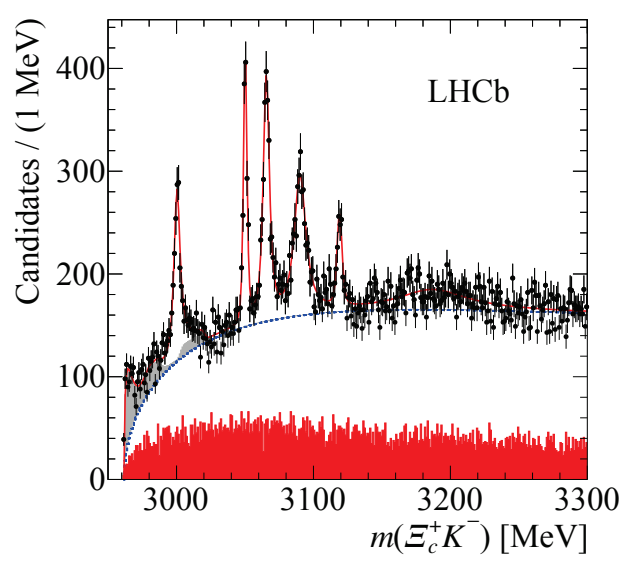

Figure 3. Discovery plot for the five narrow $\Omega_{C}$ baryons observed by LHCb. Figure taken from [33].
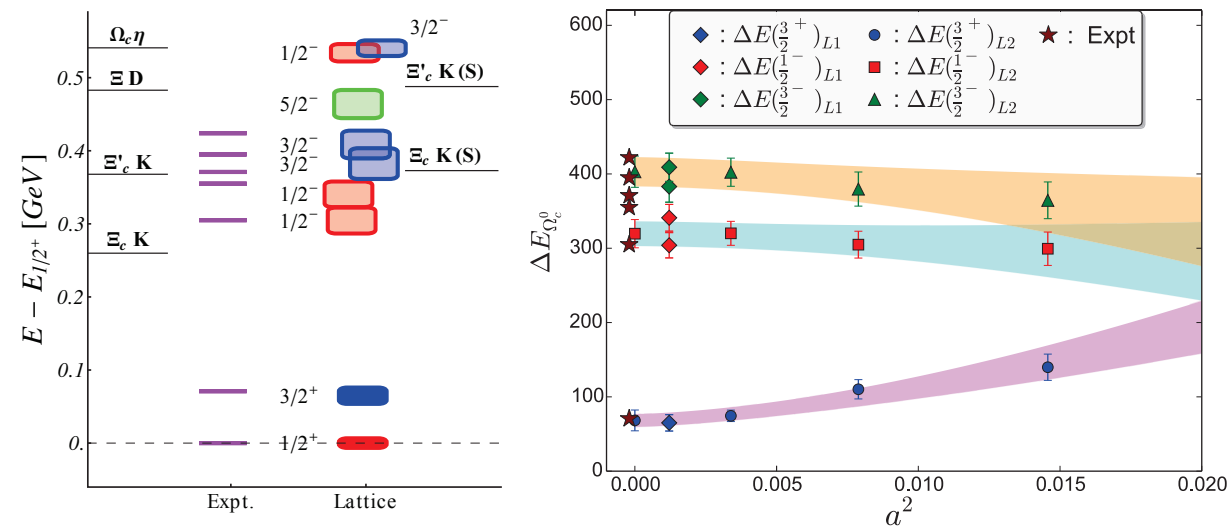

Figure 4. Left: Comparisons of energy splittings in the $\Omega_{c}$ baryon spectrum seen in experiment and the lowest energy levels determined on the lattice. For the lattice data the $J^{P}$ quantum numbers are denoted and the lowest S-wave scattering thresholds are plotted with black lines. Right: Continuum extrapolation for three of the energy levels using a different lattice action. Both Figures taken from [34].

This is done in a recent letter [34], where the pattern of excitations from two different lattice calculations is compared to the excitations observed by LHCb. In a first calculation, the authors use a large basis of interpolating fields for charmed baryons and determine the continuum spin with the method first described in [35]. The results are collected in the left pane of Figure 4, where the mass-splittings to the $J^{P}=\frac{1}{2}^{+}$ground state are compared to experiment. Their result suggests that the lowest two states observed by LHCb might have $J^{P}=\frac{1}{2}^{-}$, while the next two might have $J^{P}=\frac{3}{2}^{-}$and the last one might have $J^{P}=\frac{5}{2}^{-}$.

To check that this picture is not spoiled by large discretization effects, the authors perform another calculation using a smaller interpolator basis and multiple lattice spacings. While their basis is not large enough to resolve the closely spaced energy levels with the same 
spin-parity and the spin $\frac{5}{2}$ state, the results shown in the right pane of Figure 4 qualitatively confirms the pattern observed previously.

\section{Heavy-light hadrons}

In this section I will briefly review existing studies determining bound states and resonances of mesons with a heavy charm or bottom quark and a light quark. One of the first candidates for an exotic heavy-light hadron was the $D_{s 0}^{*}(2317)$ with $J^{P}=1^{+}$[36], whose mass is not only much lighter than expected from older potential model calculations such as [37], but also almost the same than the mass of the corresponding $J^{P}=0^{+} \mathrm{D}$-meson. Short after its discovery, it has been noted that the close-by D-meson-kaon threshold is of special importance for this state [38].

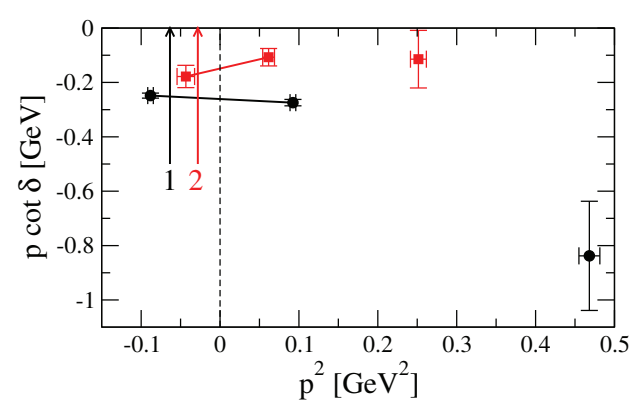

Figure 5. Determination of the scattering length and effective range used to extract the $D_{s 0}^{*}(2317)$ pole position. Ensemble (1) black circles, Ensemble (2) red squares; the vertical arrows indicate the square of the binding momenta of the bound state for ensembles (1) and (2), and the dashed line indicates the threshold. Figure from [13].

In lattice QCD the $D_{s 0}^{*}(2317)$ and the $D_{s 1}(2460)$ belonging the to same $j=\frac{1}{2}$ doublet in the heavy-quark limit have been investigated in $[12,13]$, extracting the finite volume energy spectrum with a combined basis of quark-antiquark and $D^{*}-\mathrm{K}$ interpolating fields. Using Lüscher's formula and an effective range approximation

$$
\begin{aligned}
p \cot \delta_{0}(p) & =\frac{2}{\sqrt{\pi} L} Z_{00}\left(1 ;\left(\frac{L}{2 \pi} p\right)^{2}\right) \\
& \approx \frac{1}{a_{0}}+\frac{1}{2} r_{0} p^{2},
\end{aligned}
$$

the two data points close-to threshold were used to determine the scattering length and effective range for two ensembles of gauge configurations with different lattice spacing (labeled as ensemble (1) and ensemble (2) below), pion mass, and volume. The resulting data is shown in Figure 5.

It should be stressed that this is a somewhat rough determination, as discretization effects, which are sizeable for charm-light states with the lattice action used remain uncontrolled, and the pion mass in the simulations is not quite physical. The left part of Figure 6 shows how the resulting energy splittings with respect to the spin-averaged ground states compare to experiment for the two ensembles used. Unlike results from previous lattice calculations, the statistical uncertainty is well under control and the pattern agrees qualitatively with the pattern from experiment. Indeed simple order of magnitude estimates of the discretization 
uncertainties expected within the Fermilab prescription for heavy quarks [10] suggest that discretization effects alone could account for the differences observed.
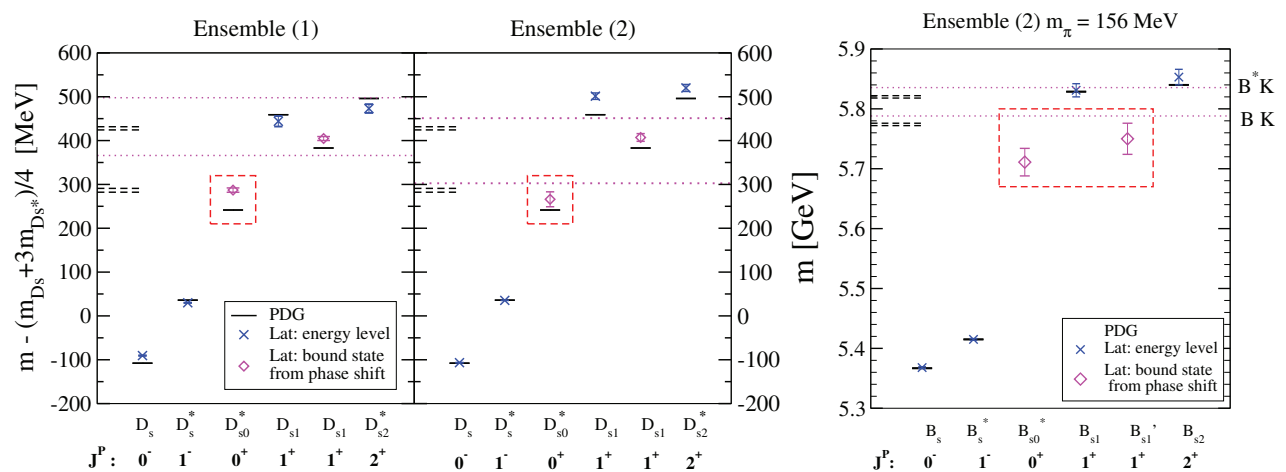

Figure 6. Left: Mass splittings in the low-lying $D_{s}$ spectrum. The lattice results for bound-state poles on Ensembles (1) and (2) are the magenta data points. Mass splittings in experiment are indicated by the black lines. Right: Mass splittings in the low-lying $B_{s}$ spectrum. The magenta data points are lattice predictions.

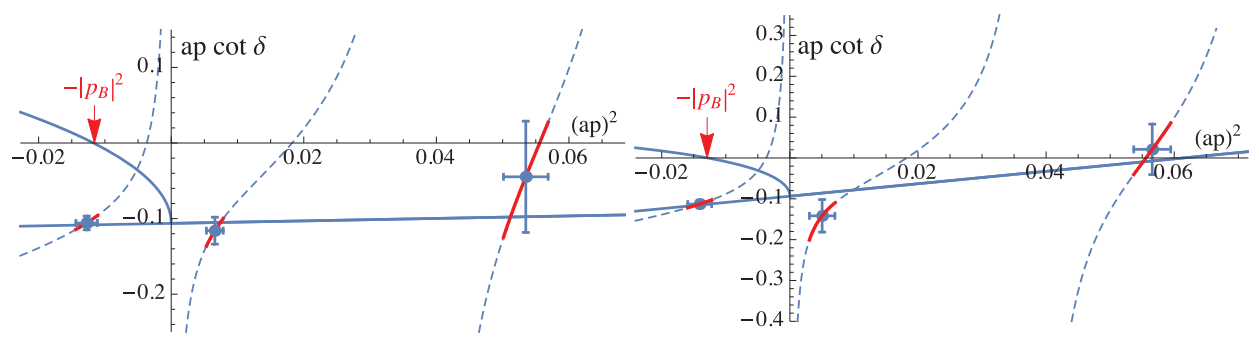

Figure 7. Effective range fits used to extract the pole positions for the predicted positive parity $B_{s}$ mesons. The left pane shows the data for S-wave scattering in the $A_{1}$ irreducible representation, while the right pane shows the $\mathrm{S}$-wave in $T_{1}$. Figure from [14]

Unlike for charm-strange mesons, the expected discretization effects for $B_{s}$ states are quite small. In [14] the positive parity $B_{s}$ cousins of the aforementioned $D_{s}$ states, which are not yet seen in experiment, have been predicted. Figure 7 shows the resulting lattice data together with an effective range approximation to the points resulting from the three lowest energy levels. The predicted spectrum is shown in the right part of Figure 6, where the uncertainties for the points in magenta now contains a full uncertainty estimate detailed in [14].

More recently, the positive parity $D_{s 0}^{*}(2317)$ and $D_{s 1}(2460)$ were revisited by different authors in [16], using lattices with multiple volumes and two different pion masses. The use of multiple lattice volumes leads to a larger number of points close-to threshold, and allows for a test of the effective range approximation, improving upon the previous results. Their results are shown in Figure 8.

The r.h.s. part of Figure 8 shows the finite volume behavior and contrasts the data from [16] with the data from one of the ensembles used in [13], and with experiment, for both the $D_{s 0}^{*}(2317)$ and the $D_{s 1}(2460)$. The extracted binding energies are roughly compatible with the 

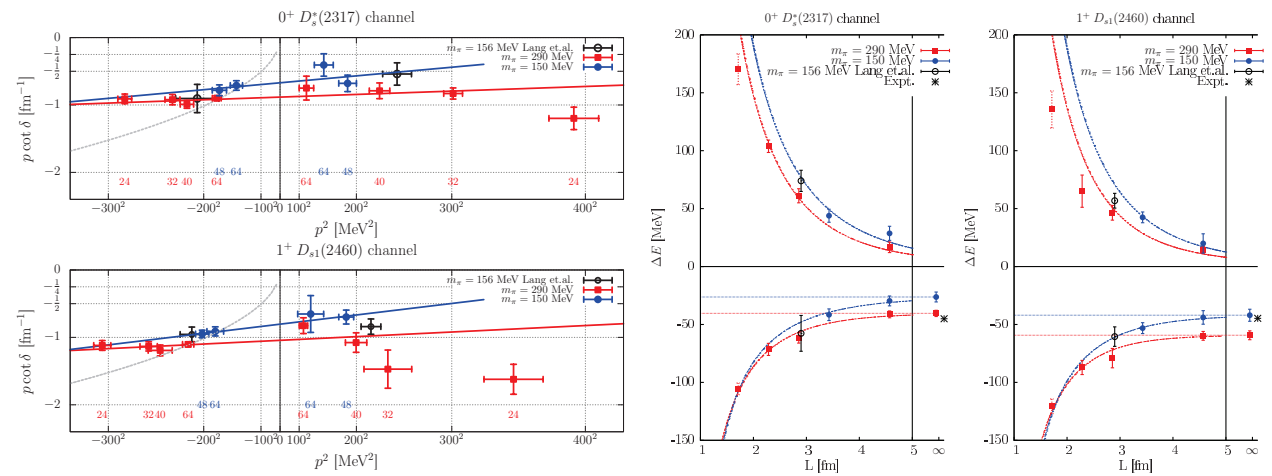

Figure 8. Left: Effective range fit used for the determination of the $D_{s 0}^{*}(2317)$ and the $D_{s 1}(2460)$ pole positions. The binding momentum of the bound state pole can be read-off from the intersection with the grey dashed line. The spatial extents $L$ of the lattices used are indicated in the Figure. Right: Finite volume behavior of the data. Figure taken from [16].

binding energies observed in experiment. It should be stressed that there has been no attempt yet to fully to control the uncertainty from the lattice discretization. Note however that the two existing lattice studies employ somewhat different methods and agree qualitatively.

Unlike for the $D_{s}$ spectrum, the $D$ mesons belonging to a heavy-quark $j=\frac{1}{2}$ doublet, the $D_{0}^{*}(2400)$ and $D_{1}(2430)$ are broad resonances in nature [1]. These have previously been studied in [11], assuming elastic $D \pi$-scattering. More recently, the Hadron Spectrum collaboration studied coupled channel $D \pi, D \eta, D_{s} K$ scattering in [15]. They employed lattice gauge configurations with multiple lattice volumes at a pion mass of $391 \mathrm{MeV}$. The use of anisotropic Wilson-clover lattices [39] allowed the determination in a number of moving frames in addition to the rest frame, which together with the multiple lattice volumes results in the the dense coverage of different center of momentum energies needed for the coupledchannel treatment.
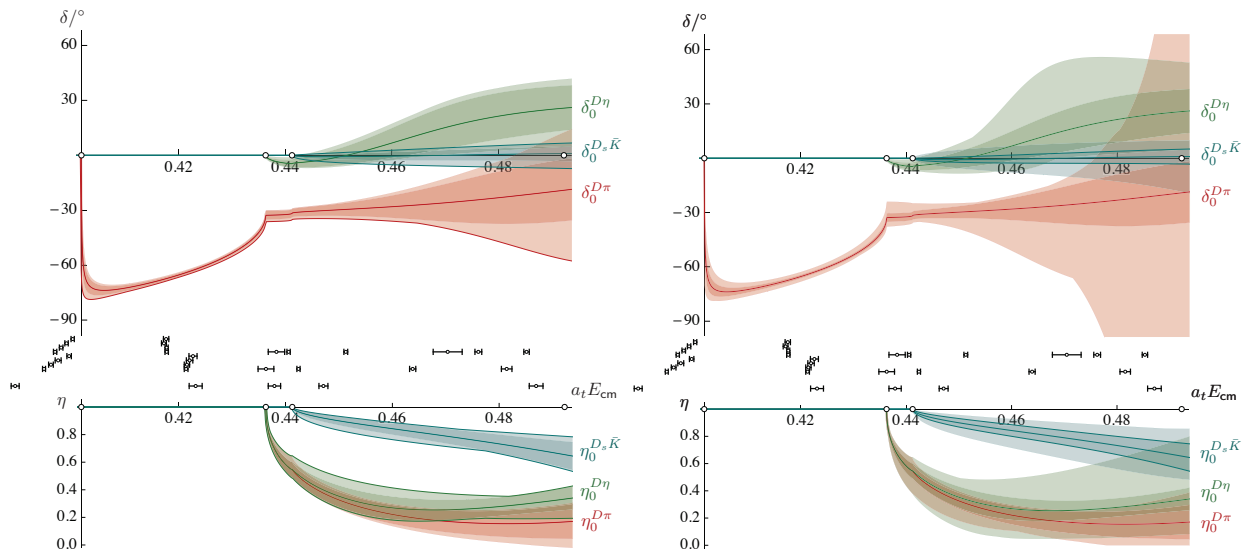

Figure 9. The 1.h.s. panes show the S-wave phase shifts and inelasticities in (coupled-channel) $D \pi, D \eta$, $D_{s} K$ scattering for a specific parameterization. The r.h.s. panes incorporate the uncertainty stemming from the choice of parameterization. Figure taken from [15]. 
They parameterize their results in terms of scattering phase shifts and inelasticities, The results of these parameterizations are shown in Figure 9. At this pion mass they observe a shallow $J^{P}=0^{+}$bound state, and a narrow $J^{P}=2^{+}$resonance. For more details please refer to $[15]$.

\section{Charmonium-like states from Lattice QCD}

Candidates for exotic mesons are most prolific in the charmonium spectrum previously believed to be well described by potential models, which made the discovery of the exotic X, Y, and $\mathrm{Z}$ charmonium-like states particularly striking. Among these, charged $Z_{c}$ states, like the $Z_{c}(3900)$ discovered by BESIII [40] and quickly confirmed by Belle [41] and using CLEO-c data [42] are most obviously of an exotic nature, as they contain a heavy charm-anticharm pair and are charged, requiring them to be made of at least 4 quarks. Nevertheless the structure of these states is heavily debated.

In [43] the finite volume spectrum with quantum numbers $I^{G} J^{P C}=1^{+} 1^{+-}$was determined using a large basis of meson-meson and four quark interpolating fields (this included tetraquark interpolators of a $3_{c} \times \overline{3}_{c}$ structure), with the aim of extracting all meson-meson states below $\approx 4.3 \mathrm{GeV}$. Unlike the studies of heavy-light mesons in the previous section, no rigorous extraction of scattering amplitudes was attempted, as the large number of open channels and the resulting high density of energy levels makes this very challenging. Furthermore, 3-particle channels were neglected completely. The strategy was instead to count the low-lying energy levels, and to identify them according to their overlaps, with the hope to see an extra-level that could be related to the presence of a $Z_{c}$ meson resonance pole.

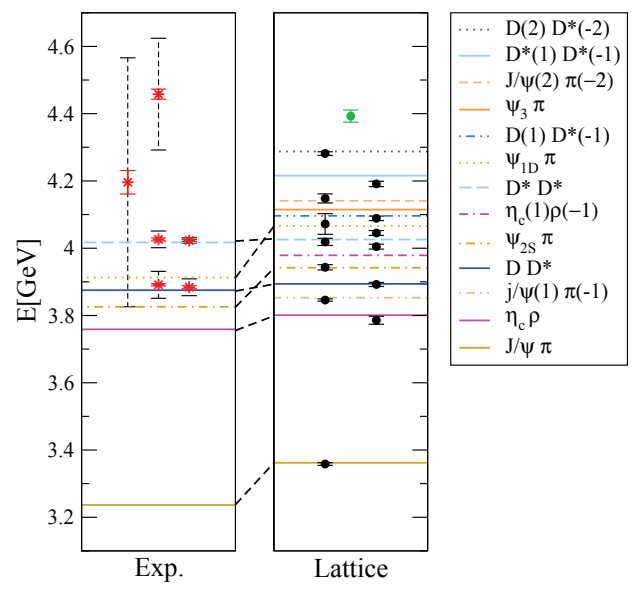

Figure 10. Left: collection of $Z_{c}$ states from experiment. The horizontal lines illustrate the various thresholds. Right: lattice spectrum compared to the non-interacting meson-meson energies (horizontal lines). Figure from [43].

The right pane of Figure 10 shows the resulting lattice spectrum compared to the noninteracting two-meson levels. The resulting spectrum is compared to observed meson states taken from [44] in the left pane. Note that no additional level is seen below $4.3 \mathrm{GeV}$, while 13 energy levels close to non-interacting meson meson energies are seen. While an additional energy level would have been a smoking-gun signature for a $Z_{c}$ state at roughly the same energy, not much can be inferred from the absence of such a level. 
A different approach is pursued in $[45,46]$, where a coupled channel extension of the HALQCD method (for the original method see [47]) is used to investigate coupled-channel scattering $J / \Psi \pi, \eta_{c} \rho, \bar{D} D^{*}$ with quantum numbers $I^{G}\left(J^{P C}\right)=1^{+} 1^{+-}$(same as above). In the HALQCD method potentials $V(r)$, functions of the distance $r$, are calculated as an intermediate quantity. The Schrödinger Equation is then solved for the given potentials and the scattering observables are extracted. For their study of coupled channel scattering in the $Z_{c}(3900)$ channel, $2+1$ flavor gauge configurations with lattice spacing $a=0.0907$ (13) and three different pion masses $m_{\pi}=410,570,700 \mathrm{MeV}$ were used.
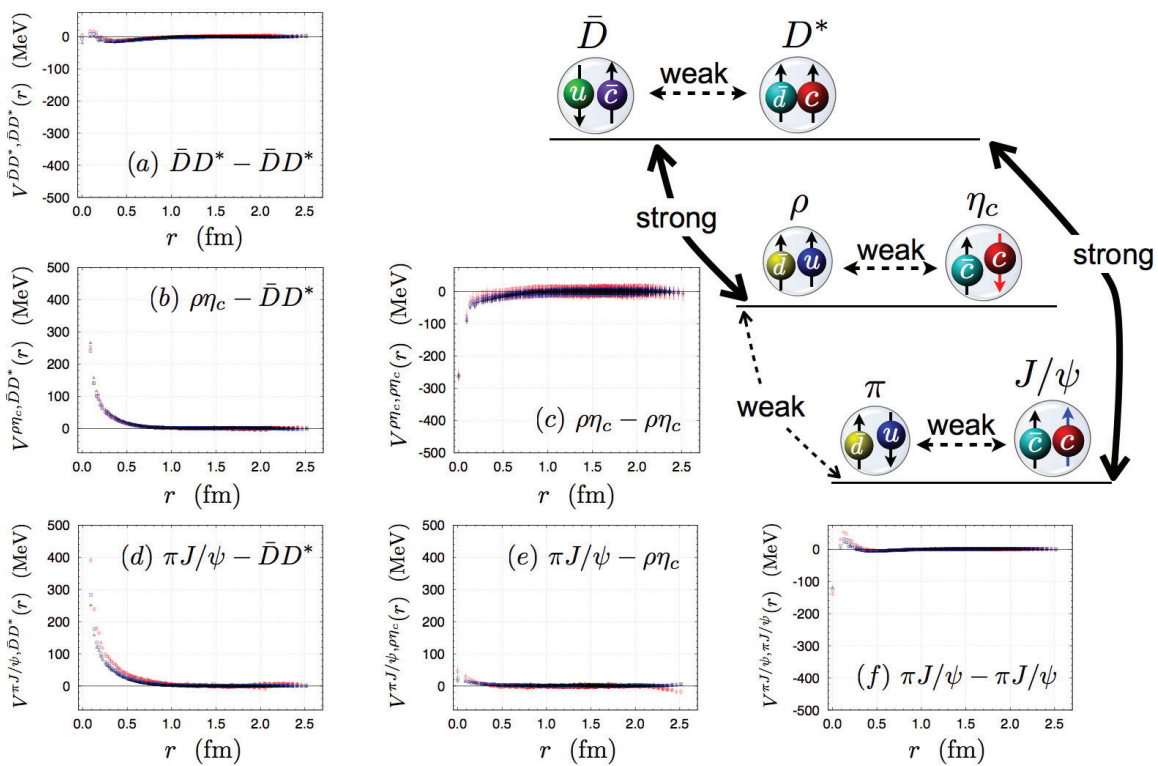

Figure 11. Coupled-channel S-wave potentials from the HALQCD method. Figure taken from [45].

Figure 11 shows the resulting diagonal and off-diagonal potentials and illustrates their pattern: While not much is happening for the diagonal, some of the off-diagonal potentials are quite strong.

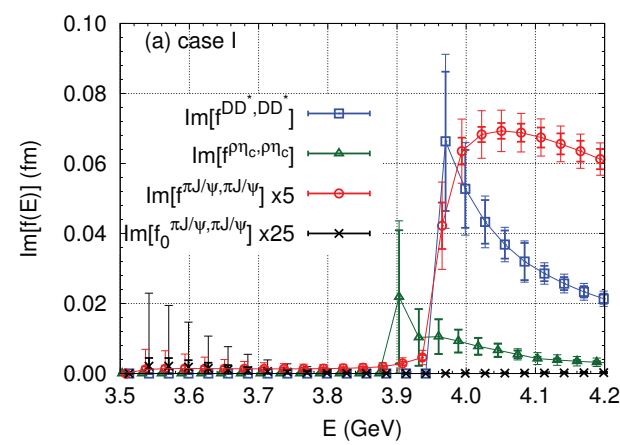

Figure 12. Two-body invariant mass spectra in the respective channels. Figure taken from [45]. 

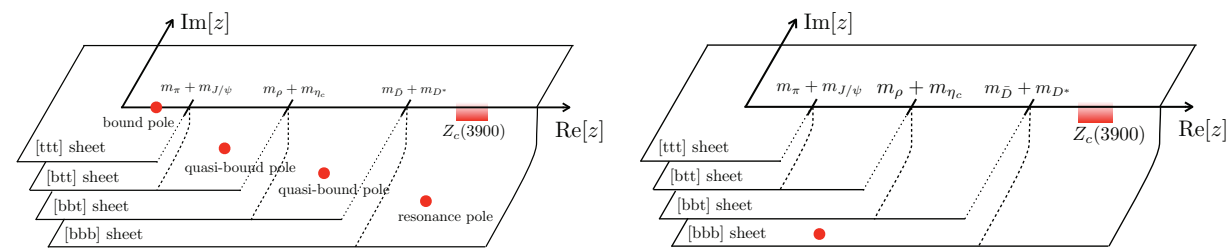

Figure 13. Left: Illustration of example pole-structures in the sheet-notation used in [46]. Right: Pole structure determined from lattice data at unphysical pion mass. Figure taken from [46].

The two-body invariant mass spectra are shown in Figure 12. The results look qualitatively similar to the pattern in experiment. Note that turning off the off-diagonal potentials instead leads to the results depicted with black crosses. The authors proceed to search for poles in the complex plane on the various Riemann sheets. For all quark masses, a pole is found far below $D D^{*}$ threshold. The pole structure is illustrated in Figure 13. The authors conclude that the $Z_{c}(3900)$ is not a usual resonance but a threshold cusp, and that the structure seen in the invariant mass comes from strong $\pi J / \Psi-\bar{D} D^{*}$ and $\rho \eta_{c}-\bar{D} D^{*}$ couplings. The authors also state that an analysis at close-to-physical pion mass is planned. Note that these results are not in obvious contradiction to the energy levels from [43].

Another interesting charmonium state is the $X(3915)$ which was previously interpreted as the first radial excitation $\chi_{c 0}^{\prime}$ of the $J^{P C}=0^{++} \chi_{c 0}$ by the PDG. This assignment was based on a determination of its quantum numbers by BaBar [48], and was heavily criticized on theoretical grounds $[49,50]$. In particular, no evidence for the fall-apart mode $X(3915) \rightarrow \bar{D} D$ has been seen, which would be expected to be the dominant decay mode for a $\chi_{c 0}^{\prime}$. Furthermore, the spin splitting $m_{\chi_{c 2}(2 P)}-m_{\chi_{c 0}(2 P)}$ between the meson in question an the well established $\chi_{c 2}^{\prime}$ is too small. Instead the $X(3915)$ is seen in the OZI suppressed decay $X(3915) \rightarrow \omega J / \psi$. Also its width should be significantly larger than $\Gamma_{\chi_{c 2}(2 P)}$. More recently, Zhou et al. [51] argued that what is dubbed $X(3915)$ is the $\chi_{c 2}(2 P)$ state already known, and just like [49] suggest that a broader state is hiding in the experiment data. More recently Belle [52] have observed a state with mass $M=3862_{-32-13}^{+26+40} \mathrm{MeV}$ and with a width of $\Gamma=201_{-067-82}^{+154+88} \mathrm{MeV}$.

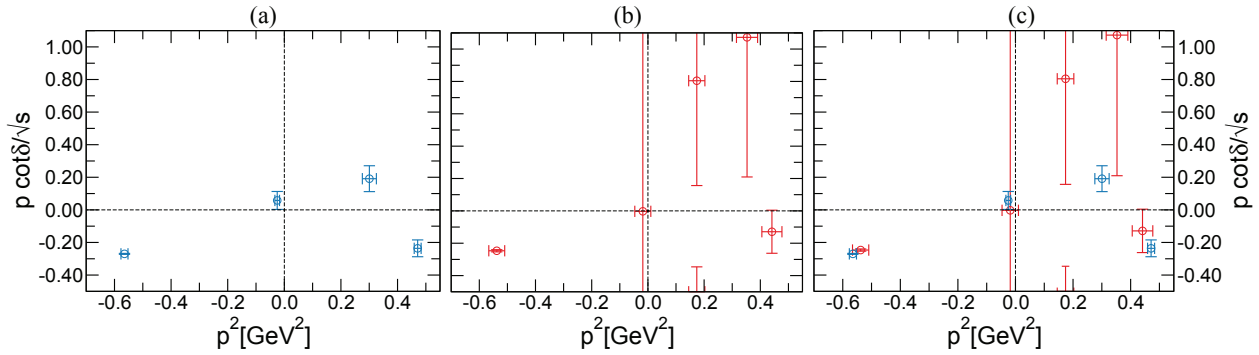

Figure 14. $p \cot \delta / \sqrt{s}$ versus $p^{2}$ scattering in S-wave. $\mathrm{p}$ is the D-meson momentum. Left: Ensemble (1); Middle: Ensemble (2); Right: Both datasets in one plot. For more details please refer to [53]

Motivated by the idea of a single broad $\chi_{c 0}^{\prime}$ resonance decaying predominantly into $\bar{D} \mathrm{D}$, the authors of [53] studied elastic $\bar{D} D$ scattering in the $J^{P C}=0^{++}$channel. Figure 14 shows the resulting data for the two ensembles used. Various possibilities are discussed in detail in [53], and the conclusion is that the lattice data suggests a fairly narrow resonance with $3.9 \mathrm{GeV} \leq M \leq 4.0 \mathrm{GeV}$ and $\Gamma<100 \mathrm{MeV}$. Notice that only the statistical uncertainty 
is quantified, and that non-negligible uncertainties stem also from the lattice discretization used and from pion mass dependence of the results. Overall neither the experiment data nor the lattice QCD results are conclusive and further results from experiment, as well as further lattice QCD results will be needed to clarify the situation.

\section{Exotic doubly-heavy hadrons}

In recent years a number of groups started to study explicitly exotic doubly-heavy hadrons using lattice QCD. In $[54,55]$ the authors studied doubly-charmed tetraquarks, while doublybottom four-quark states are studied in [56-60]. In this chapter I will highlight some of the newer results.

The authors of $[59,60]$ study B meson - B meson interactions with static bottom quarks. They determine the potentials of two static antiquarks in the presence of two light quarks on the lattice and search for bound states (rather than resonances). The potentials are calculated on lattice gauge configurations with a lattice spacing of $a=0.079 \mathrm{fm}$ and a pion mass $m_{\pi} \approx$ $650,480,340 \mathrm{MeV}$. The lattice potentials are fit with a 3-parameter form (keeping $\mathrm{p}$ fixed)

$$
V(r)=-\frac{\alpha}{r} \exp \left(-\left(\frac{r}{d}\right)^{p}\right)+V_{0}
$$

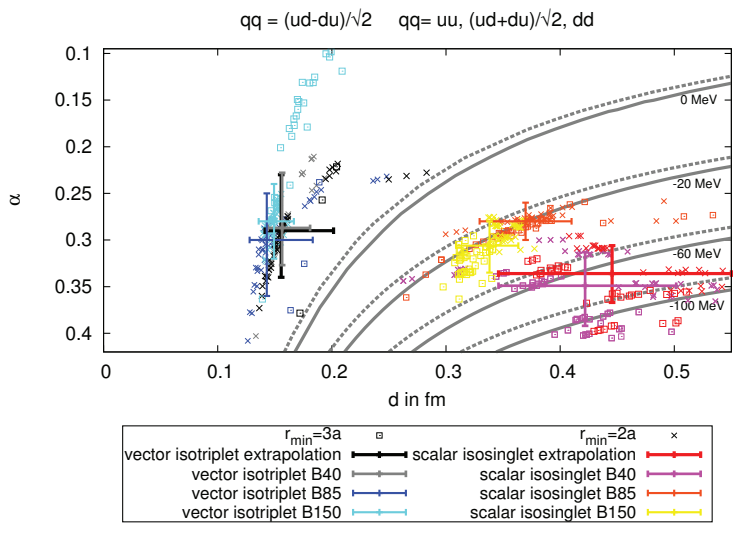

Figure 15. Binding energy isolines for the scalar isosinglet and vector isotriplet channels. Figure taken from [59].

In a first publication [59] the authors study the scalar isosinglet and vector isotriplet channels neglecting effects of the heavy $\bar{b}$ spin. Solving a non-relativistic Schrödinger equation, they find the vector isotriplet channel to be unbound, both at all three of the pion masses separately and in the limit of physical pion masses. In the scalar isosinglet case they instead observe binding for all pion masses and obtain the binding energy $90_{-36}^{+43} \mathrm{MeV}$ after a chiral extrapolation. The results from this publication are shown in Figure 15. More recently a similar procedure (solving the coupled channel Schrödinger equations) was used to also include effects of the heavy $\bar{b}$ spin [60]. A lower and somewhat less significant binding energy $E_{B}=59_{-38}^{+30} \mathrm{MeV}$ is obtained in this case.

In [61] the authors study doubly bottom $J^{P}=1^{+}$tetraquarks with NRQCD b-quarks. They us lattice gauge configurations with three different pion masses and $164 \mathrm{MeV} \leq M_{\pi} \leq$ $415 \mathrm{MeV}$ at a single lattice spacing. They obtain energy levels that suggest rather deeply 

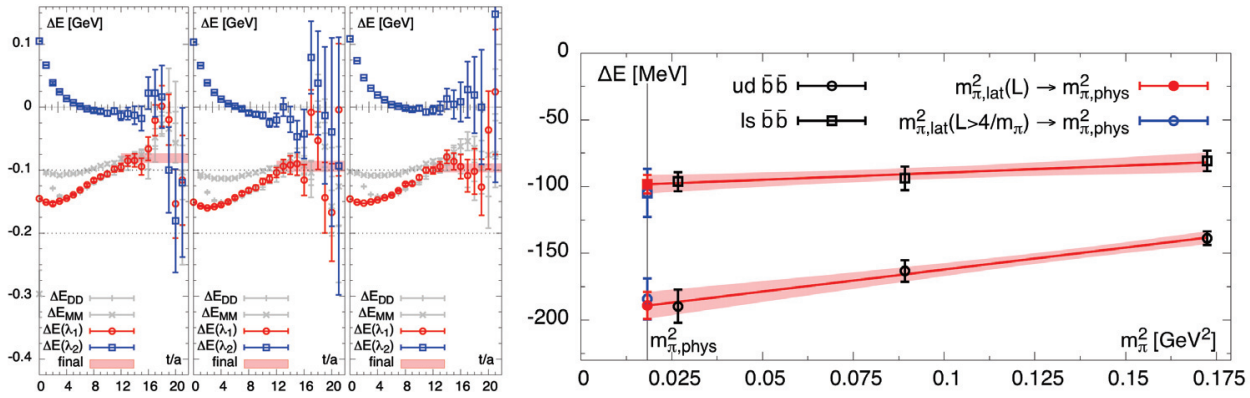

Figure 16. Left: $l s \bar{b} \bar{b}$ effective binding energies extracted from the ground state (red), and behavior for the excited state (blue). The final fit results are indicated by the red band. Right: Chiral extrapolation of the resulting binding energies. Figure taken from [61].

bound states for four-quark systems with both $u d \bar{b} \bar{b}$ and $l s \bar{b} \bar{b}$ flavor structure. The left part of Figure 16 shows the lattice energy levels they obtain and the chiral extrapolation of their results is shown in the right part of the same Figure. It should be noted that the binding energies are extracted from ratios of correlation functions. In this procedure care has to be taken [62]. A somewhat peculiar feature of the data is that the energies corresponding to the first excited state are naively expected above threshold for a bound state, however no significant shift is visible in the data. It is also expected that finite volume effects may significantly alter the observed binding energies. Given these caveats the authors quote 189(10) and 98(7) MeV as the binding energies for the $u d \bar{b} \bar{b}$ and $l s \bar{b} \bar{b}$ states respectively.
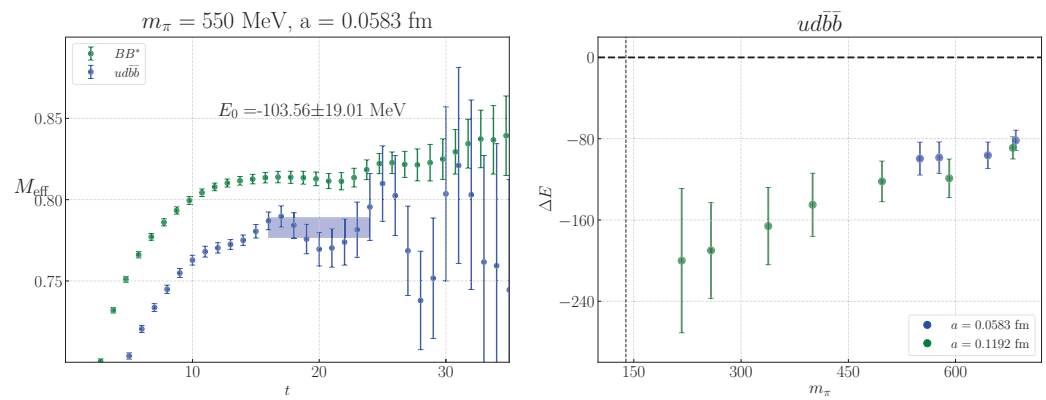

Figure 17. Effective masses of the $u d \bar{b} \bar{b}$ and (non-interacting) $B B^{*}$ system illustrating clearly that the interaction is attractive. Right: Preliminary results for the resulting binding energies. Figure taken from [63].

In [63] the authors present preliminary results for the same system using overlap valence quarks (for light, strange and charm) on staggered gauge configurations and lattice NRQCD for the bottom-quarks. For their current results they use two HISQ gauge ensemble with pion masses 305 and $319 \mathrm{MeV}$ and lattice spacings $a \approx 0.12 \mathrm{fm}$ and $a \approx 0.06 \mathrm{fm}$ respectively. On each of those ensembles they work with valence pions at various pion masses (partially quenched setup). Example results for their effective masses ares shown in the left pane of Figure 17. 
Their preliminary results at rather low statistics confirm the existence of a bound state state at heavier than physical pion mass. These results are shown in Figure 17. The binding energies at comparable quark mass agree roughly with the values presented in [61], both of which are much larger than the value obtained in the static limit in [59].

\section{Summary}

I reviewed recent progress in lattice QCD calculations of heavy hadrons. One of the highlights from recent years has been the prediction of the mass of the $\Xi_{c c}$ (and of a number of other charmed baryons) by various groups [26-32], which has been presented in Section 2.

With regard to heavy-light mesons (see Section 3), recent simulations employing Lüscher's finite volume method for the study of charmed-light mesons show a qualitative agreement with experiment $[11-13,15,16]$. To achieve this agreement, it is vital to use a diverse basis of interpolating field operators of both quark-antiquark and meson-meson structure. For bottom-strange mesons a quantitative prediction for the not yet observed positive parity $j=\frac{1}{2}$ doublet has been presented [14]. These states appear as QCD bound states in $B K$ and $B^{*} K$ scattering, and are the bottom analogues of the $D_{s 0}^{*}(2317)$ and $D_{s 1}(2460)$.

In Sections 4 and 5 exploratory results for exotic charmonia and doubly-heavy hadrons have been presented. Among these, evidence for the existence of doubly-heavy bound states with flavor content $u d \bar{b} \bar{b}$ and $l s \bar{b} \bar{b}[59,61,63]$ is especially intriguing.

\section{Acknowledgements}

I would like to thank Parikshit Junnarkar and Nilmani Mathur for sending me (at the time of the talk) unpublished material. I would also like to thank C. B. Lang and M. Padmanath for their careful reading of the manuscript.

\section{References}

[1] S. Aoki et al., Eur. Phys. J. C77, 112 (2017), 1607.00299

[2] S. Borsanyi et al., Science 347, 1452 (2015), 1406.4088

[3] M. Lüscher, Nucl. Phys. B354, 531 (1991)

[4] M. Lüscher, Nucl. Phys. B364, 237 (1991)

[5] K. Rummukainen, S.A. Gottlieb, Nucl. Phys. B450, 397 (1995), hep-lat/9503028

[6] R.A. Briceno, J.J. Dudek, R.D. Young (2017), 1706.06223

[7] M. Peardon, J. Bulava, J. Foley, C. Morningstar, J. Dudek, R.G. Edwards, B. Joo, H.W. Lin, D.G. Richards, K.J. Juge (Hadron Spectrum), Phys. Rev. D80, 054506 (2009), 0905.2160

[8] C. Morningstar, J. Bulava, J. Foley, K.J. Juge, D. Lenkner, M. Peardon, C.H. Wong, Phys. Rev. D83, 114505 (2011), 1104 . 3870

[9] A.X. El-Khadra, A.S. Kronfeld, P.B. Mackenzie, Phys. Rev. D55, 3933 (1997), hep-lat/9604004

[10] M.B. Oktay, A.S. Kronfeld, Phys. Rev. D78, 014504 (2008), 0803.0523

[11] D. Mohler, S. Prelovsek, R.M. Woloshyn, Phys. Rev. D87, 034501 (2013), 1208.4059

[12] D. Mohler, C.B. Lang, L. Leskovec, S. Prelovsek, R.M. Woloshyn, Phys. Rev. Lett. 111, 222001 (2013), 1308.3175

[13] C.B. Lang, L. Leskovec, D. Mohler, S. Prelovsek, R.M. Woloshyn, Phys. Rev. D90, 034510 (2014), 1403.8103 
[14] C.B. Lang, D. Mohler, S. Prelovsek, R.M. Woloshyn, Phys. Lett. B750, 17 (2015), 1501.01646

[15] G. Moir, M. Peardon, S.M. Ryan, C.E. Thomas, D.J. Wilson, JHEP 10, 011 (2016), 1607.07093

[16] G.S. Bali, S. Collins, A. Cox, A. Schäfer, Phys. Rev. D96, 074501 (2017), 1706.01247

[17] R. Aaij et al. (LHCb), Phys. Rev. Lett. 119, 112001 (2017), 1707.01621

[18] M. Mattson et al. (SELEX), Phys. Rev. Lett. 89, 112001 (2002), hep-ex/0208014

[19] A. Ocherashvili et al. (SELEX), Phys. Lett. B628, 18 (2005), hep-ex/0406033

[20] S.J. Brodsky, F.K. Guo, C. Hanhart, U.G. Meissner, Phys. Lett. B698, 251 (2011), 1101.1983

[21] S.P. Ratti, Nucl. Phys. Proc. Suppl. 115, 33 (2003), [,33(2003)]

[22] B. Aubert et al. (BaBar), Phys. Rev. D74, 011103 (2006), hep-ex/0605075

[23] R. Chistov et al. (Belle), Phys. Rev. Lett. 97, 162001 (2006), hep-ex/0606051

[24] Y. Kato et al. (Belle), Phys. Rev. D89, 052003 (2014), 1312 . 1026

[25] R. Aaij et al. (LHCb), JHEP 12, 090 (2013), 1310.2538

[26] C. Alexandrou, V. Drach, K. Jansen, C. Kallidonis, G. Koutsou, Phys. Rev. D90, 074501 (2014), 1406.4310

[27] R.A. Briceno, H.W. Lin, D.R. Bolton, Phys. Rev. D86, 094504 (2012), 1207.3536

[28] Z.S. Brown, W. Detmold, S. Meinel, K. Orginos, Phys. Rev. D90, 094507 (2014), 1409.0497

[29] Y. Namekawa et al. (PACS-CS), Phys. Rev. D87, 094512 (2013), 1301. 4743

[30] P. Pérez-Rubio, S. Collins, G.S. Bali, Phys. Rev. D92, 034504 (2015), 1503.08440

[31] S. Mondal, M. Padmanath, N. Mathur, Spectroscopy of Charmed and Bottom Hadrons using Lattice QCD, in 35th International Symposium on Lattice Field Theory (Lattice 2017) Granada, Spain, June 18-24, 2017 (2017), 1712 . 08446, http: //inspirehep . net/record/1644787/files/arXiv: 1712.08446.pdf

[32] R. Lewis, N. Mathur, R.M. Woloshyn, Phys. Rev. D64, 094509 (2001), hep-ph/0107037

[33] R. Aaij et al. (LHCb), Phys. Rev. Lett. 118, 182001 (2017), 1703.04639

[34] M. Padmanath, N. Mathur, Phys. Rev. Lett. 119, 042001 (2017), 1704.00259

[35] J.J. Dudek, R.G. Edwards, M.J. Peardon, D.G. Richards, C.E. Thomas, Phys. Rev. Lett. 103, 262001 (2009), 0909.0200

[36] B. Aubert et al. (BaBar), Phys. Rev. Lett. 90, 242001 (2003), hep-ex/0304021

[37] S. Godfrey, N. Isgur, Phys. Rev. D32, 189 (1985)

[38] E. van Beveren, G. Rupp, Phys. Rev. Lett. 91, 012003 (2003), hep-ph/0305035

[39] H.W. Lin et al. (Hadron Spectrum), Phys. Rev. D79, 034502 (2009), 0810. 3588

[40] M. Ablikim et al. (BESIII), Phys. Rev. Lett. 110, 252001 (2013), 1303.5949

[41] Z.Q. Liu et al. (Belle), Phys. Rev. Lett. 110, 252002 (2013), 1304.0121

[42] T. Xiao, S. Dobbs, A. Tomaradze, K.K. Seth, Phys. Lett. B727, 366 (2013), 1304.3036

[43] S. Prelovsek, C.B. Lang, L. Leskovec, D. Mohler, Phys. Rev. D91, 014504 (2015), 1405.7623

[44] N. Brambilla et al., Eur. Phys. J. C74, 2981 (2014), 1404. 3723

[45] Y. Ikeda, S. Aoki, T. Doi, S. Gongyo, T. Hatsuda, T. Inoue, T. Iritani, N. Ishii, K. Murano, K. Sasaki (HAL QCD), Phys. Rev. Lett. 117, 242001 (2016), 1602.03465

[46] Y. Ikeda (HAL QCD) (2017), 1706.07300

[47] N. Ishii, S. Aoki, T. Hatsuda, Phys. Rev. Lett. 99, 022001 (2007), nucl-th/0611096

[48] J.P. Lees et al. (BaBar), Phys. Rev. D86, 072002 (2012), 1207. 2651 
[49] F.K. Guo, U.G. Meissner, Phys. Rev. D86, 091501 (2012), 1208. 1134

[50] S.L. Olsen, Phys. Rev. D91, 057501 (2015), 1410.6534

[51] Z.Y. Zhou, Z. Xiao, H.Q. Zhou, Phys. Rev. Lett. 115, 022001 (2015), 1501.00879

[52] K. Chilikin et al. (Belle), Phys. Rev. D95, 112003 (2017), 1704.01872

[53] C.B. Lang, L. Leskovec, D. Mohler, S. Prelovsek, JHEP 09, 089 (2015), 1503.05363

[54] Y. Ikeda, B. Charron, S. Aoki, T. Doi, T. Hatsuda, T. Inoue, N. Ishii, K. Murano, H. Nemura, K. Sasaki, Phys. Lett. B729, 85 (2014), 1311.6214

[55] A.L. Guerrieri, M. Papinutto, A. Pilloni, A.D. Polosa, N. Tantalo, PoS LATTICE2014, 106 (2015), 1411.2247

[56] Z.S. Brown, K. Orginos, Phys. Rev. D86, 114506 (2012), 1210. 1953

[57] P. Bicudo, M. Wagner (European Twisted Mass), Phys. Rev. D87, 114511 (2013), 1209.6274

[58] P. Bicudo, K. Cichy, A. Peters, B. Wagenbach, M. Wagner, Phys. Rev. D92, 014507 (2015), 1505.00613

[59] P. Bicudo, K. Cichy, A. Peters, M. Wagner, Phys. Rev. D93, 034501 (2016), 1510.03441

[60] P. Bicudo, J. Scheunert, M. Wagner, Phys. Rev. D95, 034502 (2017), 1612 . 02758

[61] A. Francis, R.J. Hudspith, R. Lewis, K. Maltman, Phys. Rev. Lett. 118, 142001 (2017), 1607.05214

[62] T. Iritani et al., JHEP 10, 101 (2016), 1607.06371

[63] P. Junnarkar, M. Padmanath, N. Mathur, Heavy light tetraquarks from Lattice QCD, in 35th International Symposium on Lattice Field Theory (Lattice 2017) Granada, Spain, June 18-24, 2017 (2017), 1712.08400, http://inspirehep.net/record/ 1644795/files/arXiv: $1712.08400 . p d f$ 